\title{
Psychophysics for Perception of (In)determinate Art
}

\author{
Christian Wallraven ${ }^{1}$, Kathrin Kaulard ${ }^{1}$, Cora Kürner $^{1}$, Robert Pepperell ${ }^{2}$, Heinrich H. Bülthoff ${ }^{1}$ \\ ${ }^{1}$ Max Planck Institute for Biological Cybernetics, Tübingen, Germany \\ ${ }^{2}$ University of Wales Institute, Cardiff, UK
}

a)
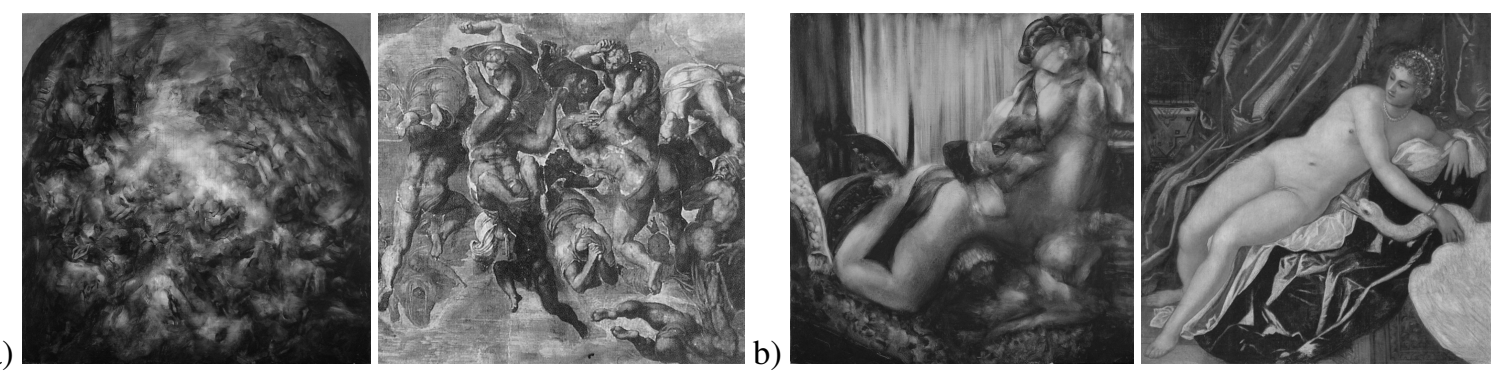

Figure 1: Abstract or representational? The left image in a) and b) shows an indeterminate painting, whereas the right image in a) and b) shows a representational painting with seemingly similar image content.

\begin{abstract}
The question of how humans perceive art and how the sensory percept is endowed with aesthetics by the human brain has continued to fascinate psychologists and artists alike. It seems, for example, rather easy for us to classify a work of art as either "abstract" or "representational". The artist Robert Pepperell recently has produced a series of paintings that seek to defy this classification: his goal was to convey "indeterminancy" in these paintings - scenes that at first glance look like they contain an object or belong to a certain genre but that upon closer examination escape a definite determination of their contents. Here, we report results from several psychophysical experiments using these artworks as stimuli, which seek to shed light on the perceptual processing of the degree of abstraction in images. More specifically, the task in these experiments was to categorize a briefly shown image as "abstract" or "representational". Stimuli included Pepperell's paintings each of which was paired with a similar representational work of art from several periods and several artistic genres. The results provide insights into the visual processes determining our perception of art and can also function as a "objective" validation for the intentions of the artist.
\end{abstract}

CR Categories: J.5 [Computer Application]: Arts and Humanities-Fine arts; J.4 [Computer Application]: Social and Behavioural Sciences-Psychology;

Keywords: perception of art, categorization, psychophysics, perceptual graphics

\section{Introduction}

Perception and processing of our surroundings seem to be so effortless and easy that we rarely pay attention to how we see. Our brain, however, has to process, combine and separate shapes, colors, shadows, object relations, and much more within fractions of a

Copyright $(92007$ by the Association for Computing Machinery, Inc.

Permission to make digital or hard copies of part or all of this work for personal or classroom use is granted without fee provided that copies are not made or distributed for commercial advantage and that copies bear this notice and the full citation on the first page. Copyrights for components of this work owned by others than ACM must be honored. Abstracting with credit is permitted. To copy otherwise, to republish, to post on servers, or to redistribute to lists, requires prior specific permission and/or a fee. Request permissions from Permissions Dept, ACM Inc., fax +1 (212) 869-0481 or e-mail permissions @acm.org.

APGV 2007, Tübingen, Germany, July 26-27, 2007.

(C) 2007 ACM 978-1-59593-670-7/07/0007 $\$ 5.00$ second in order to build a coherent representation from sensory input - it is only this representation that allows us to interact with our environment. Visual illusions demonstrate that this process of interpretation often does not correspond to physical reality, but rather that perception critically depends on heuristics, or, more generally, prior knowledge. It is this realization of the truth of "perceptual reality" that underlies the works of the artist Robert Pepperell. Using different techniques, he tries to create works of art that evoke the impression of "something" but that at a closer look escape a definite interpretation of what that "something" consists of (see Figure 1). This type of "indeterminate" art is made possible by playing with visual features that are perceptually important for object and scene interpretations - shapes that seem to consist of coherent patches and fragments of color, but that stop just short of defining the object or scene completely.

Pepperell's interest in "indeterminate" art arose from viewing a scene in an expressionist movie (Robert Wienes 'The Cabinet of Dr. Caligari' (1919)) whose visual content could for a short time not be interpreted fully - stayed indeterminate: "I was no longer a passive consumer of meaning, but an active producer, drawing on all my latent cognitive resources to recover the lost sense of coherence..." [Pepperell 2005]. Following this realization, Pepperell started to work on producing art that captures this moment of indeterminacy: "When trying to construct such images I soon realized the difficulties inherent in balancing the right amount of meaningfulness and meaninglessness so as to create the desired indeterminate effect. If it was too meaningless then it attracted no interest at all; if it was too meaningful then there was no anxiety about the depiction and, hence, no effect of the kind I was seeking." [Pepperell 2005] He goes so far as to say that "The world itself contains no objects [...] the world out there is simply a unitary mass of potential objects that, with the right apparatus, can be perceived as separate, bounded things.'[Pepperell 2005]

Pepperell's art in a way occupies the middle ground between "abstract" and "representational" art - wavering between being devoid of meaning and determining a clear genre and image content. In this paper, we want to look at Pepperell's art with the tools of a perception scientist - more specifically, we are interested in how we process indeterminate art and whether categories such as "abstract" and "representational" are categories that are available perceptually. We will shed light on how we perceive indeterminate art and in doing so also provide a perceptual validation of Pepperell's 


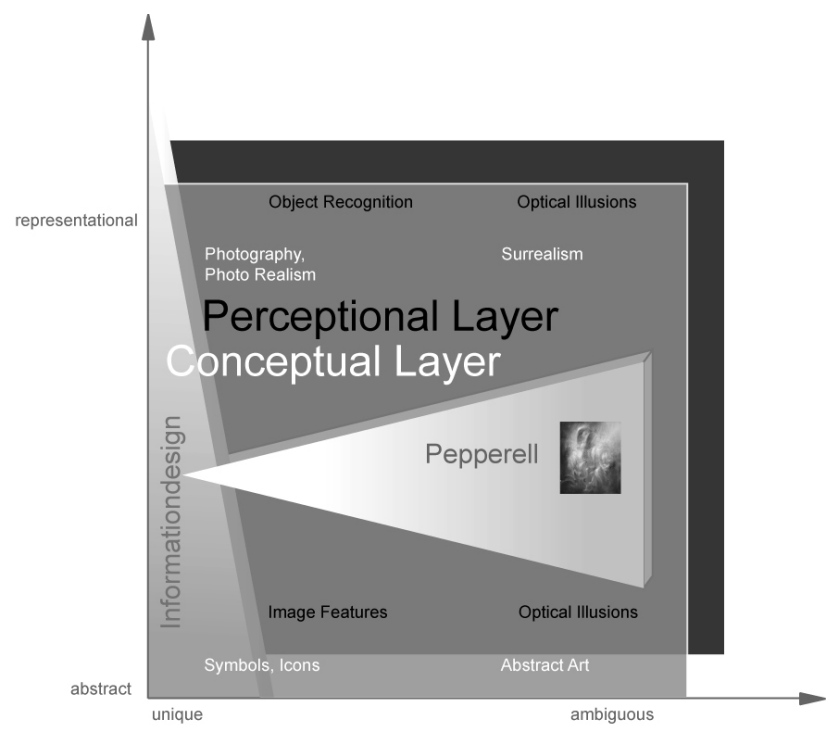

Figure 2: The connection between art, perception, and visualization using the two parameter dimensions of "abstract"/"representational" and "unique"/"ambiguous".

artistic intentions, as we will be able to determine for each painting whether it succeeded in wavering between "abstract" and "representational". At the same time, Pepperell's paintings give us as perception scientists a unique set of stimuli with which we can test and probe the processes underlying scene categorization by constraining and determining, for example, which features give rise to visually well-defined objects and scenes.

Going beyond a connection between art and perception research, it is perhaps interesting in this context to take a look at visualization or information design: An effective visualization, for example, needs to be as clearly and uniquely interpretable as possible. The creation of effective visualizations can therefore be aided by looking at perceptual processes: "[...] information design must actively encourage and participate in research that increases our understanding of information and the effect that it has: how and why people respond to information, how the human brain processes information and builds knowledge, as well as how humans organize knowledge and convert it into improved behavior and operation. Better understanding of these factors will enable us to create the best possible information, interfaces and communications." [Knemeyer 2003].

The connection between these topics is illustrated by Fig. 2 which represents a schematic space consisting of two broad interpretation dimensions (abstract/representational and unique/ambiguous), as well as two broad instantiation layers of visual information (information can be interpreted at the higher, conceptual layer and the lower, perceptual layer). For each of the extremes in this twodimensional space and for each layer, we have indicated "typical" applications - for example, on the perceptual layer the goal of object recognition as a fundamental process of our visual system is to unambiguously interpret real-world objects; the equivalent on the conceptual layer is given by photography or photo-realism in computer graphics which are concerned with an accurate depiction of the physical world.

It is obvious that the conceptual layer is always dependent on and defined by the perceptual layer showing that one needs to integrate both levels for information design/visualization and, for example, indeterminate art. On the conceptual layer, the parameters of abstract/representational and unique/ambiguous allow the assign- ments of different visual rendering techniques as well as their underlying perceptual equivalents. The uniqueness that is intended in information design and visualization is illustrated by its positioning on the left of the diagram. Pepperell's art, however, needs to be found in the ambiguous domain of this scale. Fig. 2 shows that perception can quickly lose its degree of uniqueness and that this can be used on the conceptual level by, for example, abstract, cubist, surrealist, or indeterminate art: the dissolution of perspective laws, the loss of shape- and color-information, as well as the presentation in different contexts all render images more ambiguous. In contrast, photography needs to be clearer in its message, similarly to information design, as "[...] information only has value when it is successfully communicated. If it cannot be accessed or understood it does not have value." [Knemeyer 2003]. Information design and visualization, in comparison to photography, try to convey complex, sometimes highly abstracted information therefore placing these disciplines firmly in the abstract and unique domain.

\section{Related Work}

There have been numerous studies that have looked at the perception of art, with approaches ranging from neurophysiology [Zeki 1999], to behavioural studies using eyetracking [Buswell 1935; Yarbus 1967], to higher-level perceptual discussions [Arnheim 1974; Fourquet et al. 2006]. To our knowledge, however, there have been no studies that have investigated works of art using psychophysical methods which try to establish a more rigorous description of the visual features that drive perception of these works. Here, we were interested in exploring the indeterminate art by Robert Pepperell in comparison to selected representational art from such a psychophysical point of view.

In the following, we will discuss two perceptual experiments that look at

- Q1: the distribution of categorical decisions between "abstract" and "representational" for quickly presented images of Pepperell's art and representational art

- Q2: how fast it takes to make this decision

- Q3: whether we process both types of art independently of their image orientation

- Q4: how the pattern of responses changes as a function of image size

The motivations for addressing these specific research questions arose from research in scene categorization and object recognition:

- ad Q1: It is known that humans can decide extremely quickly and accurately whether a scene contains an animal [Thorpe et al. 1996]; similarly, the gist of a scene is available to us by just taking a quick glance at a picture [Potter 1975; Biederman et al. 1981]. Can we also use "high-level" categories such as "abstract" and "representational" in such a context?

- ad Q2: Connected to the previous question: does the processing of clearly defined image contents take as long as the processing of indeterminate features in Pepperell's works?

- ad Q3: Numerous studies have demonstrated that perception is critically dependent on stimulus transformations (see, for example, the discussion on view-based recognition in the object recognition literature [Tarr and Bülthoff 1999], or the face inversion effect [Yin 1969]). By presenting indeterminate and representational art in different image orientations, we hope to get insight into whether the processing of these two categories is driven by low-level, highly orientation-independent features, or rather higher-level, orientation-dependent features. 

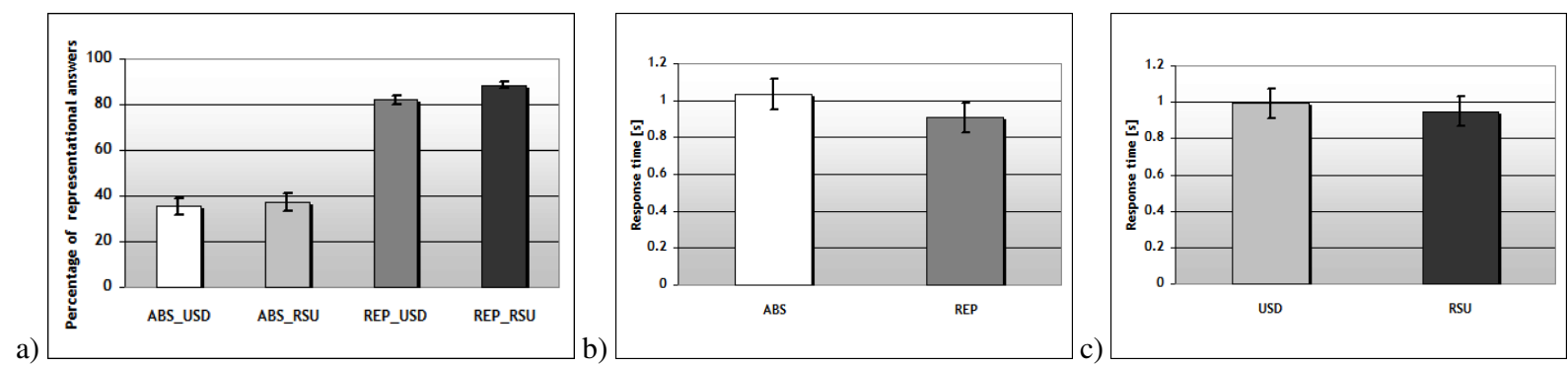

Figure 3: Category responses as a function of a) category and orientation. Reaction times as a function of b) category, c) orientation.

- ad Q4: Similarly, the question of scale is important in visual processing: face detection, for example, using only lowfrequency information is better in the context of a scene than without this context [Lewis and Edmonds 2003]. It will be interesting to see, how we process these high-level concepts of "abstract" and "representational", as a function of image size as this determines the critical spatial frequencies for this task.

\section{Experiment 1}

In this experiment, we addressed questions Q1-Q3 using 60 artworks from Robert Pepperell (some examples are shown in Figs. $6,7,8)$ and 60 representational artworks. The latter were selected by Robert Pepperell as they could be seen as representional counterparts to or inspiration for his art. They were by different artists and represented rather different genres and styles. All images were converted to greyscale - therefore, in the following experiments, we explicitly do not address the role of color in the perception of art.

\subsection{Methods and Design}

Fifteen participants were shown 60 images of indeterminate art and 60 images of representational art. In order to address the role of image orientation images were shown three times in the correct (right-side-up, RSU) orientation and three times rotated by $180^{\circ}$ (upside-down, USD) yielding a total of 720 trials. Images were shown on a monitor at a resolution of $1024 \times 768$ pixels which participants viewed from a distance of $50 \mathrm{~cm}$ while resting their head on a chin rest. As the paintings were of different formats, image size varied accordingly from $500 \times 500$ pixels to $700 \times 600$ pixels - on average, images subtended $\approx 20^{\circ} \times 20^{\circ}$ of visual angle. Each trial started with a fixation cross that was displayed for $100 \mathrm{~ms}$, after this an image was displayed for $120 \mathrm{~ms}$, followed by a blank period of $300 \mathrm{~ms}$. Starting with the image presentation, participants had to answer using two keys, whether the image was "abstract" or "representational" - they were told to do this as quickly as possible and the time from image onset to keypress was recorded as their reaction time. Participants were briefed before the experiments about those two categories and no participant had any problems with these concepts. This task can be seen as probing the abstract/representational interpretation dimension depicted in Fig. 2 for Pepperell's art.

\subsection{Analysis}

Analysis of the results was done by subjecting the variables category response and reaction time to an analysis of variance with factors category and orientation. For category response, we found main effects of category $(\mathrm{F}(1,14)=7.572, \mathrm{p}<0.05)$ and orientation $(\mathrm{F}(1,14)=11.321, \mathrm{p}<0.01)$ as well as a highly significant interaction between category and orientation $(\mathrm{F}(1,14)=16.371, \mathrm{p}<0.001)$. For reaction time, we found effects of category $(\mathrm{F}(1,14)=10.758$, $\mathrm{p}<0.01)$ and orientation $(\mathrm{F}(1,14)=10.633, \mathrm{p}<0.01)$.

\subsubsection{Categorization}

The results for category response are plotted in Fig. 3a as the percentage of trials in which participants responded that a particular image would belong to the representational category.

Category: The category describes the two types of art - indeterminate art versus representational art. The analysis shows that overall, $63 \%$ of the participants labelled Pepperell's images as "abstract" meaning that $37 \%$ saw a known object or scene in those images. This result already tells us that Pepperell's intentions of indeterminacy have largely been achieved in his images - ideally, of course, the result would have been $50 \%$. We will discuss these results in more detail below for some individual images. In contrast, representational images were categorized as such in $85 \%$ of the cases - a much more unambiguous, but still not perfect categorization result. Nevertheless, especially this latter result shows that participants are, indeed, capable of reliably assigning high-level concepts to briefly shown images. This demonstrates that going beyond the scene gist, higher-level concepts may be accessible already at a very early stage in the perceptual process.

Category/Orientation: The comparison of right-side-up versus upside-down images shows that representational images were significantly better recognized as such in the upright condition $(88 \%$ versus $82 \%$, $(\mathrm{p}<0.01))$. This result provides further evidence for the theory that we process scenes as configurations of objects, and that these configurational processes are critically dependent on viewing direction (see also [Vogel et al. 2006] for further studies on configurational processing of real-world scenes). Conversely, the lack of an orientation effect for indeterminate art might suggest that either those images are not processed on an object level but rather more globally, or that these types of images do lack a defined image composition with clear directionalities and image relations.

Single Images: Looking at performance for single images, we see a more refined pattern: out of 60 images, 44 representational images were recognized equally well in both orientations (see Figs. 6a,b for two examples) - it was never the case, however, that an upside-down image was recognized better than a right-side-up image. Interestingly, several of the images, for which no effect of inversion was found, depicted ceiling frescos (such as shown in Fig. 6a,b) which show human figures in various arrangements containing faces in all orientations. This would make it much easier for the human visual system - which is known to be orientation sensitive for detection [Lewis and Edmonds 2003] and recognition [Wallraven et al. 2002] of faces - to analyze these images despite a change in orientation. A significant inversion effect could be found for 16 representational images. It is interesting that in this case, the images predominately contain low special frequencies resulting in a slightly blurred, unfocussed look (see Figs. 6c,d for two examples). In Fig. 6d, for example, one can recognize stairs in the left side of the image. By using the prior that light in natural scenes comes from above [Brewster 1826], the pattern of light and dark makes features, which in isolation would be rather obscure, more easily interpretable. Turning this image upside down, this interpretation suddenly becomes much harder due to the apparently random distribution of light and 
shadows in the scene thus resulting in a more abstract impression. For Pepperell's images, the two images that were classified as being most "representational" are shown in Figs. 6e,f (scores for these images were $86 \%$ and $76 \%$, respectively). Looking more closely at these images, Fig. 6e might depict a reclining figure on a dais whereas Fig. $6 \mathrm{f} \mathrm{might} \mathrm{depict} \mathrm{a} \mathrm{bedouin} \mathrm{figure} \mathrm{in} \mathrm{an} \mathrm{oriental} \mathrm{bazaar}$ scene. Both images share a very clear delineation of shapes with relatively sharp boundaries and high-frequency information. The images with the highest number of "abstract" votes are shown in Fig. 6g,h (scores for these images were $3 \%$ and 5\%, respectively). These two paintings (as well as several other very "abstract" ones) consist mostly of blurred, unfocussed shapes with few - if any - coherent regions that could be identified as objects. Interestingly, Fig. $6 \mathrm{~h}$ also shows a very clear inversion effect in that it is categorized as significantly more representational in the upside-down condition. This shows that this image (as well as two other of Pepperell's artworks which we tested) seem to contain a configuration of image features that appears more well-defined in the inverse orientation. Image 22 shows an inversion effect in that it is classified as more "abstract" when turned upside-down (a change from 52\% to $70 \%$ ) - here, Pepperell seems to leave his intended domain of indeterminate art. This image (as well as 6 additional images for which this effect could be found) contains two parts: a relatively busy, highcontrast region compared to a quiet, low-contrast region. This latter region could be interpreted as background and/or sky. The shapes on the opposite side therefore need to specify the lower part of the image - again, our prior knowledge tells us that objects are usually on the ground with light shining on them from above. This defined orientation seems to enable a more representational interpretation of the image therefore leading to an inversion effect.

\subsubsection{Reaction Time}

The results for category response are shown in Figs. 3b,c. Category: Participants were consistently faster to categorize representational images than they were for indeterminate art. One explanation for this might be that participants use specific search strategies for this task - the search for known objects in the scene, for example, in Pepperell's images by intention would be unsuccessful therefore leading to longer reaction times. This result is especially interesting in the context of the category responses discussed above: if the strategy for participants in the categorization task would simply be to look for "blurred" images and classify those as "abstract" then one would presumably find a much faster reaction time for these images, as low spatial frequencies can be processed much faster (e.g, [Lewis and Edmonds 2003]). As this was not the case, we can assume that categorization in both cases is driven predominately by the search for known objects and object relations in the scene - this result agrees with previous studies on scene categorization [Biederman et al. 1981; Henderson 2005].

Orientation: Overall, we found that images in the right-side-up condition were categorized faster than images in the upside-down condition. This was true for both categories, showing that even though category response for Pepperell's images did not depend on orientation, reaction time did. Again, this result demonstrates that there seems to be a defined composition of objects or object-like features in Pepperell's images and that these are processed differentially depending on orientation.

\section{Experiment 2}

In Experiment 2, we were interested to address the question of scale (Q4) in the categorization task in order to investigate how the assignment of the high-level concepts of "abstract" and "representational" would depend on image size. For this, we used three different image sizes created by successively reducing the images by a factor of 2 arriving at three sizes of $1 / 2$ original size, 1/4 original size, and 1/8 original size. In addition, all images were shown only once in those three sizes - again using upside-down and rightside-up orientations. The task, methods, and design were otherwise identical to Experiment 1 with another group of 15 participants taking part in this experiment.

\subsection{Analysis}

Analysis of the results was done by subjecting the variables category response and reaction time to an analysis of variance with factors category, orientation, and size. For category response, we found main effects of category $(\mathrm{F}(1,14)=9.893, \mathrm{p}<0.01)$ and size $(\mathrm{F}(2,28)=18.758, \mathrm{p}<0.001)$ as well as a highly significant interactions between category and orientation $(\mathrm{F}(1,14)=26.292, \mathrm{p}<0.001)$ and category and size $(\mathrm{F}(2,28)=15.788, \mathrm{p}<0.001)$. For reaction time, we also found main effects of category $(\mathrm{F}(1,14)=10.852$, $\mathrm{p}<0.01)$ and orientation $(\mathrm{F}(1,14)=5.376, \mathrm{p}<0.05)$, as well as an interaction between category and size $(\mathrm{F}(2,28)=5.405, \mathrm{p}<0.01)$.

\subsubsection{Categorization}

Category: The overall difference between indeterminate and representational images was much bigger than in the first experiment: $52 \%$ of all indeterminate images were categorized as "abstract", whereas $77 \%$ of all representational images were labelled as such. Presenting images at smaller scales seemingly has brought Pepperell's art closer to being indeterminate. Similarly, there was a significant reduction for representational images - again, reducing image size led to a more abstract percept.

Category/Orientation: Looking at the interaction of category and orientation for representational artworks, right-side-up images were categorized as such in $81 \%$ as opposed to only $74 \%$ of the cases in the upside-down condition - a significant difference $(\mathrm{p}<0.01)$. In contrast, performance did not change much overall for Pepperell's artworks. This result is in accordance with Experiment 1 where we found the same pattern.

Category/Size: Not surprisingly, the bigger the images, the better categorization becomes for representational images rising from $65 \%$ for the lowest scale to $81 \%$ for the middle scale, to $87 \%$ for the highest scale. Interestingly, the performance for the largest scale in this experiment was identical to the performance in Experiment 1 $(\mathrm{p}=0.65, \mathrm{n} . \mathrm{s})$ where the images were twice as large. A further increase in size beyond the scale tested here therefore seems not to help any further in labelling the images as "representational" - at larger image sizes, this might also be due to the time it takes to scan the image using eye movements as retinal image size becomes too big. Nevertheless, a reduction to a quarter of the original image size (corresponding to an average image size of $170 \times 170$ pixels) already results in a noticable drop in performance, whereas a reduction to an eighth of the original image size (corresponding to an average image size of $85 \times 85$ pixels) has a detrimental effect on performance suggesting that the critical information for this task is contained at this spatial frequency range.

For Pepperell's paintings, the degree of representationality ranges from $42 \%$ to $51 \%$ - although there is a tendency for smaller images to be classified as more "abstract" than large images, this difference does not reach statistical significance ( $\mathrm{p}>0.05$, n.s. for all comparisons). Nevertheless, there is a significant difference of the largest scale in this experiment to be judged as more "abstract" than the one used in Experiment 1 (45\% versus 36\%, $(\mathrm{p}<0.01)$ collapsed across orientations). It therefore seems that the scale at which indeterminacy is achieved is on average at half the original image size for the viewing distance used here - at least when defining indeterminacy along the dimension of representationality as was done for these experiments.

Single images: For representational images, the images which exhibited the largest difference across scales are shown in Figs. 7a,b 

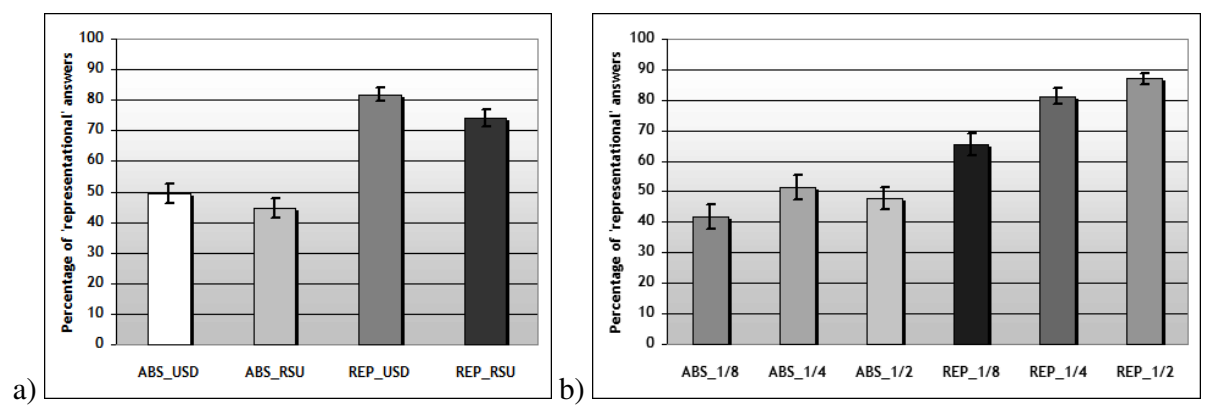

Figure 4: Category responses as a function of a) category and orientation, d) category and size.

(the scores across scales were 40\%, 80\%, 93\% for Fig. 7a and 13\%, $60 \%, 80 \%$ for Fig 7b). The large increase in performance for both images for the middle scales almost represents a categorical change in perception as the image contents become recognizable. Conversely, Fig. 7c shows an example from the 20 images for which a change in scale had almost no effect on categorization performance. These images usually contain larger objects (such as the human figures in Fig. 7c) that remain clearly visible even at a much smaller image scale. Interestingly, comparing and combining the results for Experiment 1 and Experiment 2, we found that representational images which result in a strong inversion effect (for example, Fig. $6 c, d)$ were also the ones which suffered most from a change in size. This might hint at a possible interaction between scale and orientation, which we could, however, not verify due to lack of statistical power using this data.

For Pepperell's indeterminate artworks, the interpretation of single images is much less clear due to the relatively small effect of scale on the results. For 10 indeterminate paintings out of 60 , we found that they were classified as being more representational with increasing size (Fig 7e with scores of $40 \%, 67 \%, 87 \%$ and Fig $7 \mathrm{f}$ with scores of $33 \%, 53 \%, 80 \%$ ). These images contained a relatively large amount of high-frequency content compared to the other indeterminate artworks. We could not find clear evidence for the inverse effect, that is images that were classified as more "abstract" with increasing size. Overall, it therefore seems that we have found a clear tendency for small image sizes to be judged as more "abstract".

\subsubsection{Reaction Time}

Category \& Orientation: Similar to Experiment 1, representational images were categorized faster than indeterminate ones right-side-up images were also categorized faster than images in the upside-down condition. This again supports the assumption that these images are processed using a search strategy for known objects and object relations.

Category/Size: Interestingly, for representational images, reaction times were faster for larger images than for smaller ones. If one assumes a search for objects for a successful classification, then this search might be easier for the larger scales as objects - such as faces, human figures or trees - are more clearly identifiable. In contrast, for indeterminate art reaction times are tendentially faster for the larger scales than for the smaller ones. Again, assuming a similar search strategy this can be explained by the fact that larger images still contain hints of objects (that is, more high-frequency information), therefore leading to a longer, yet still largely unsuccessful search ultimately resulting in longer reaction times.

\section{Genre categorization}

After a short break, we asked participants for both experiments to do another task. Using the same experimental setup, we randomly presented 30 images of indeterminate art in the original size on the screen and asked participants to categorize each image into one of 6 categories or artistic genres (Biblical scene, Landscape with person, Landscape without person, Portrait, Stillife, Battle Scene) or to indicate a new category. Participants could take as long as they wanted to make this decision. Our goal here was to see whether participants would be able to assign a category to an indeterminate image - again, if Pepperell's intentions would be true, we would expect few if any consistent answers in this task. This therefore task probes the unique/ambiguous dimension on Fig. 2.

\subsection{Uniqueness criterion}

In order to determine the validity of the categorization reponses across images, we use a uniqueness criterion that is defined as:

$$
u=\frac{1}{5 \cdot \sum_{j=1}^{n} v_{j}} \cdot \sum_{i=1}^{n}\left(v_{\max }-v_{i}\right)
$$

that is, for each image, we determine the category with the highest number of votes $v_{\max }$ and then sum up the differences between this maximum value and the votes for all $n=6$ categories normalizing by $n-1=5$ times the total number of votes. An image with a large uniqueness criterion has a value of 1 , whereas a criterion of 0 would mean a maximally ambiguous image. Note, that this definition does not take into account whether participants chose to select a different category from the 6 given ones - across both experiments, this was done in $15 \%$ of the cases.

In the following, we present the results of this analysis collapsed across both experiments - there were slight differences between the two experiments which could, however, still be explained by variation due to the relative coarseness of the uniqueness measure.

\section{2 "Unique" and "Ambiguous"}

Fig. 8 shows the three most uniquely and the two most ambiguously classified images according to the uniqueness criterion.

Uniquely categorized images: Fig. 8a was the most uniquely categorized image with 28 out of 30 participants labelling it as a stilllife. Looking at the painting, this categorization can clearly be verified both the composition as well as single "object" (bananas, an apple) can be seen in the image. Similarly, Fig. $8 \mathrm{~b}$ was also classified as a stilllife by 16 participants (most of the remaining participants saw flowers in the image); one might say that the image can perhaps best be interpreted as a painting of a flower stillife as the broad, paste-like brush-strokes of this style can be clearly identified in the image - even if the individual objects cannot. Finally, Fig. 8c was categorized as a portrait by 21 participants - again, an interpretation which seems "reasonable" as the image reminds of a painting of a reclining figure on a dais.

Ambiguously categorized images: Figs. 8d,e seemingly could not be categorized consistently at all - except for the genre of portrait all other genres were named for these three images. Participants were at least unanimous in that all those images did not con- 
Interpretation Space

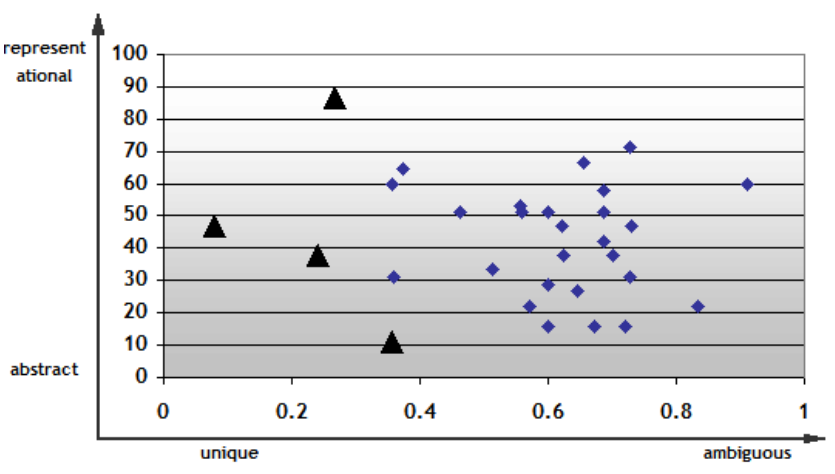

Figure 5: Pepperell's interpretation space.

tain a close view of a person; interestingly, however, the category of Landscape containing person(s) was chosen regularly indicating that there might have been enough information to assume the presence of human figures in the image. Some of the shapes in Fig. 8d, for example, might consist of human figures/shadows in the mist.

\section{The interpretation space - Conclusions}

Coming back to the questions raised in the introduction, we have found evidence that

- ad Q1: Participants are able to assign high-level concepts such as "abstract" and "representational" to briefly presented images. Performance for representational art was around 90\% for upright images indicating a very robust processing pipeline. Indeterminate art was classified as lying on the middle of the representationality dimension providing initial validation for Pepperell's artistic intentions.

- $\operatorname{ad} \mathrm{Q} 2$ : The time it makes to do this decision is slightly longer for indeterminate art than for representational art. This is a strong indication that this task is solved by a search strategy looking for known objects and object relations.

- ad Q3: We found a clear impact of orientation on processing of representational art that was in accordance with previous research on our sensitivity to stimulus transformations in visual processing. This was even true to some extent for indeterminate artworks which still contained remnants of standard image composition techniques (light region above dark region).

- ad Q4: Finally, we found that processing of the degree of abstractness critically depends on the image size, that is, the spatial frequency content of the images. The smaller the images were in Experiment 2, the more "abstract" they appeared to be - again, taking reaction time data into account, this speaks in favour of a search strategy.

In the following, we sum up our results in the context of the interpretation space developed in the introduction. By using the two dimensions of "abstract"-"representational" and "unique""ambiguous" which we measured using the results from the fast categorization experiment and from the genre catgorization data, we can assign two coordinates to each image and therefore locate it in the interpretation space (see Fig. 5).

It is interesting that the images occupy a large part of the interpretation space - no clear correlation between the two dimensions could be found indicated by the best-fit score that was non-significant $\left(r^{2}=0.052, \mathrm{p}=0.45, \mathrm{n} . \mathrm{s}\right)$. This result can be seen as a validation of the concept behind Fig. 2 which locates art, perception and visualization in this two-dimensional space. As hypothesized earlier, Pepperell's images, indeed, occupy a region of indeterminacy mostly lying in the "ambiguous" region and that his images in addition fall inbetween abstract and representational concepts with a slight tendency towards the abstract. Following a strict interpretation of this space, the four images marked on the left could be seen as lying outside of Pepperell's original intentions as they are too uniquely determinable along either of the two dimensions. In a way, these results can therefore be seen as a perceptual validation of Pepperell's artistic programme - if only a very crude one.

Further work will be concerned with a better characterization of the spatial frequency information contained in the images. We intentionally chose not to include this here, as we want to combine this analysis with a set of eye-tracking experiments that are currently underway. Taken together, these experiments and analyses aim at characterizing more rigourously the visual information that is used in such a categorization task following up on the classic work of [Buswell 1935; Yarbus 1967]. Finally, we will analyze the role of color in the perception of both indeterminate and representational art as well as include truly "abstract" artworks in order to be able to span "all of the interpretation space".

\section{References}

ARNHEIM, R. 1974. Art and visual perception: a psychology of the creative eye: the new version. University of California Press.

Biederman, I., Mezzanotte, R. J., Rabinowitz, J. C., Francolini, C. M., AND Plude, D. 1981. Detecting the unexpected in photointerpretation. Hum Factors 23, 2 (Apr), 153-164.

BREWSTER, D. 1826. On the optical illusion of the conversion of cameos into intaglios, and of intaglios into cameos, with an account of other analogous phenomena. Edinburgh Journal of Science 4, 99-108.

Buswell, G. 1935. How people look at pictures: A study of the psychology of perception in art. Univ. Chicago Press, Chicago.

Fourquet, E., Cowan, W., And Mann, S. 2006. Multiple perspectives in computer graphics: arguments from perceptual grouping and renaissance art. In $A P G V$ '06: Proceedings of the 3rd symposium on Applied perception in graphics and visualization, ACM Press, New York, NY, USA, 154-154.

Henderson, J., E. 2005. Visual Cognition.

KNEMEYER, D. 2003. Information design: Information design the understanding discipline. Online BoxesAndArrows.

LEWIS, M., AND EDMONDS, A. 2003. Face detection: Mapping human performance. Perception 32, 902-920.

Pepperell, R. 2005. Visual indeterminacy and the paradoxes of consciousness. In Proceedings of Altered States Conference.

PotTer, M. 1975. Meaning in visual scenes. Science 185, 165-166.

TARr, M., AND Bülthoff, H. 1999. Object Recognition in Man, Monkey, and Machine (Cognition Special Issues). Cambridge, USA: MIT Press.

Thorpe, S., Fize, D., AND MARlot, C. 1996. Speed of processing in the human visual system. Nature 381, 520522.

Vogel, J., Schwaninger, A., Wallraven, C., And Bülthoff, H. H. 2006. Categorization of natural scenes: local vs. global information. In APGV '06: Proceedings of the 3rd symposium on Applied perception in graphics and visualization, ACM Press, New York, NY, USA, 33-40.

Wallraven, C., Schwaninger, A., Schumacher, S., And Bülthoff, H. 2002. View-based recognition of faces in man and machine: Re-visiting interextra-ortho. In Biologically Motivated Computer Vision, Springer, H. Bülthoff, S.W. Lee, T. Poggio, and C. Wallraven, Eds., vol. 2525 of Lecture Notes in Computer Science, 651-660.

YARBUS, A. 1967. Eye movements and vision. Plenum Press, New York.

YIN, R. 1969. Looking at upside-down faces. Journal of Experimental Psychology.

ZEKI, S. 1999. Inner vision: an exploration of art and the brain. Oxford University Press. 

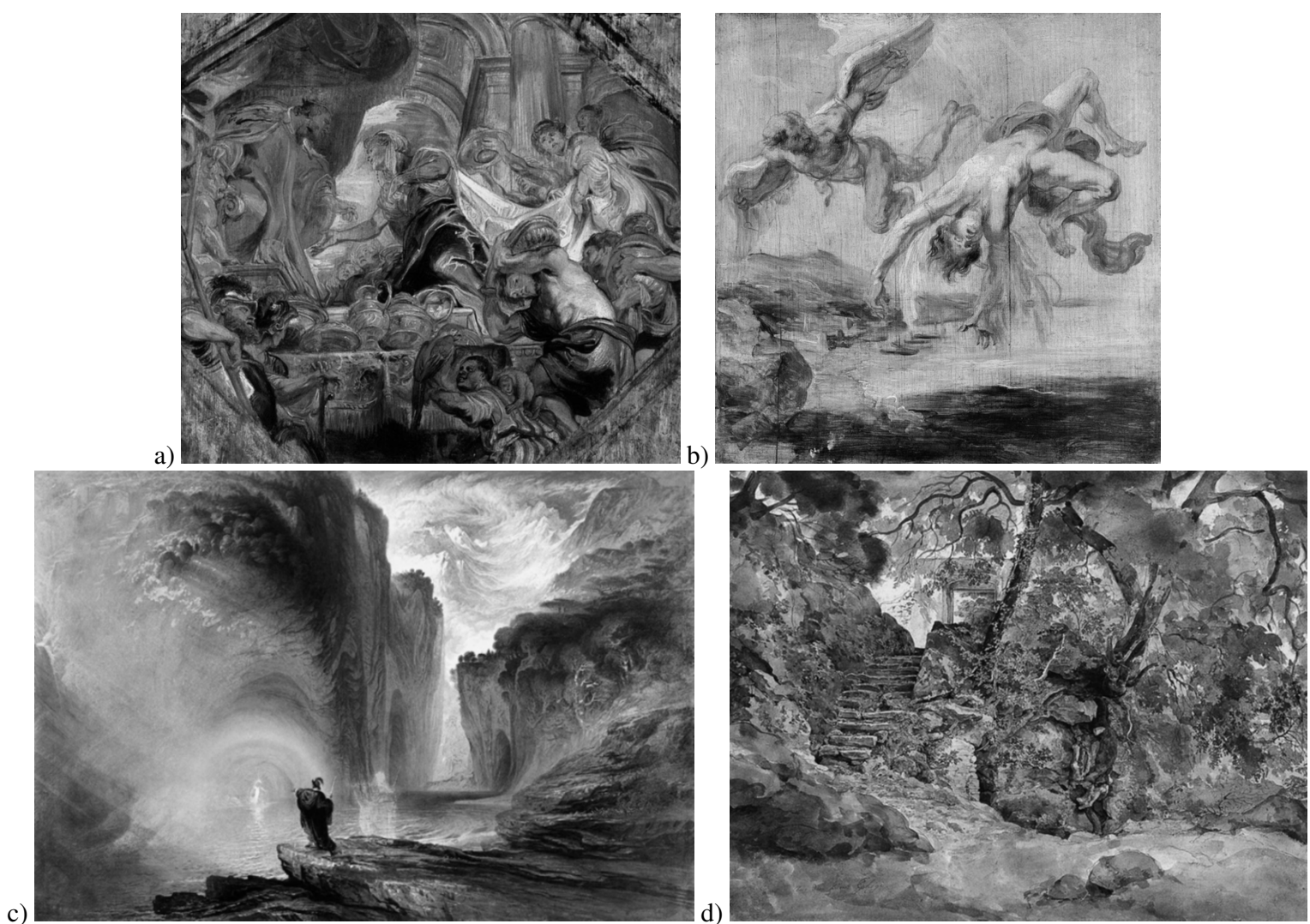

c)
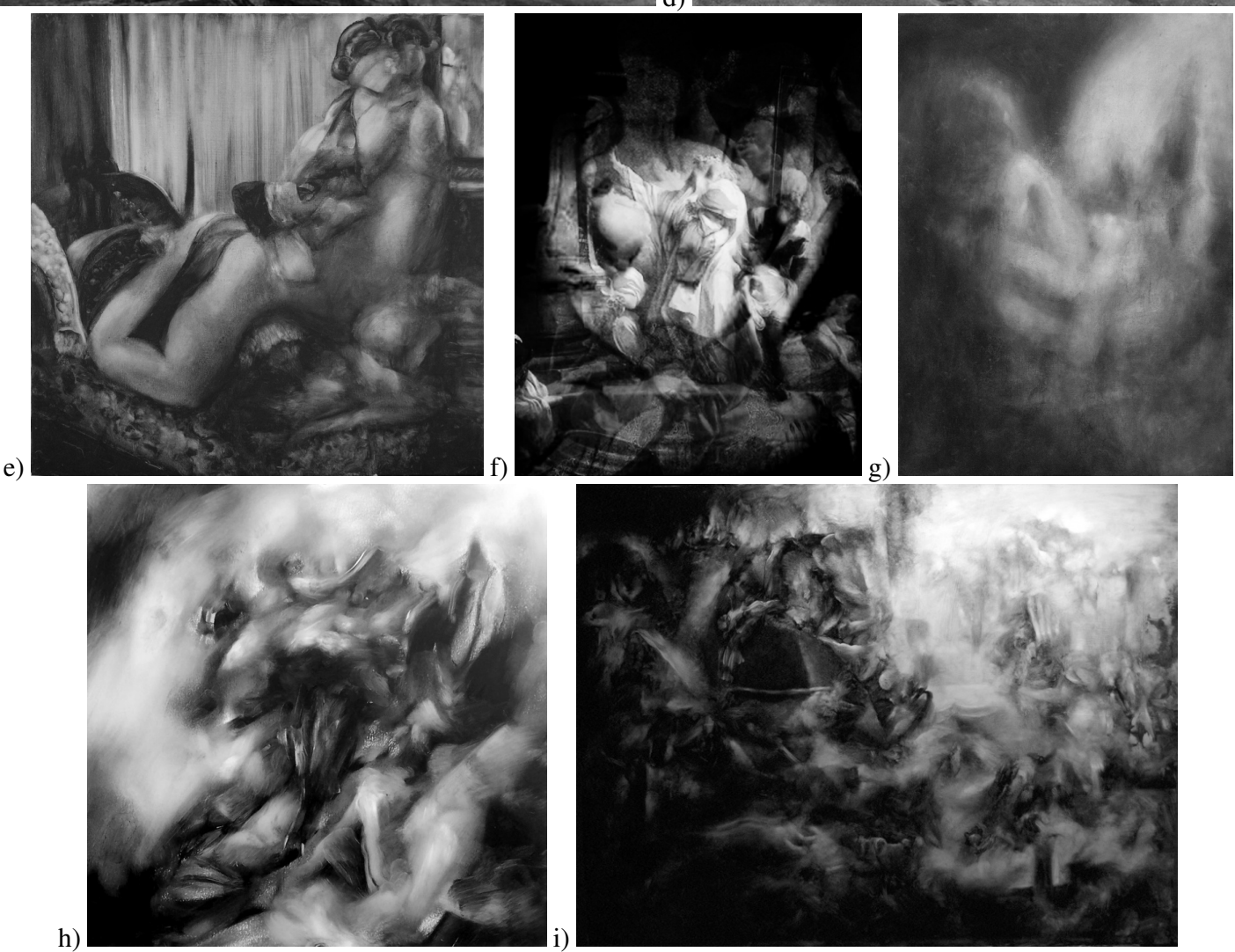

Figure 6: Exp1: a)-d) Examples of representational art, e)-i) examples of indeterminate art by Robert Pepperell. 

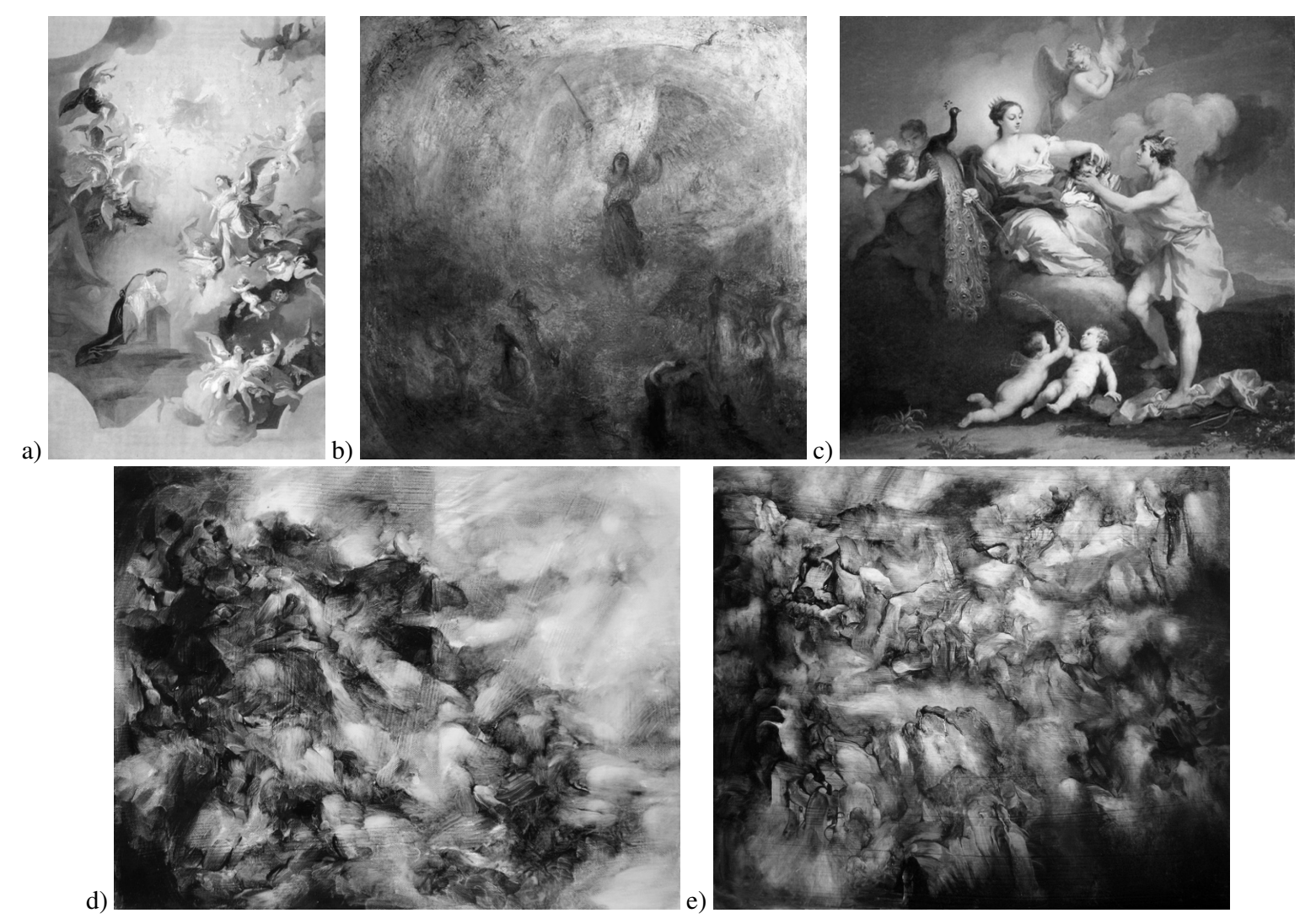

Figure 7: Exp2: a)-c) Examples of representational art, d)-e) examples of indeterminate art by Robert Pepperell.
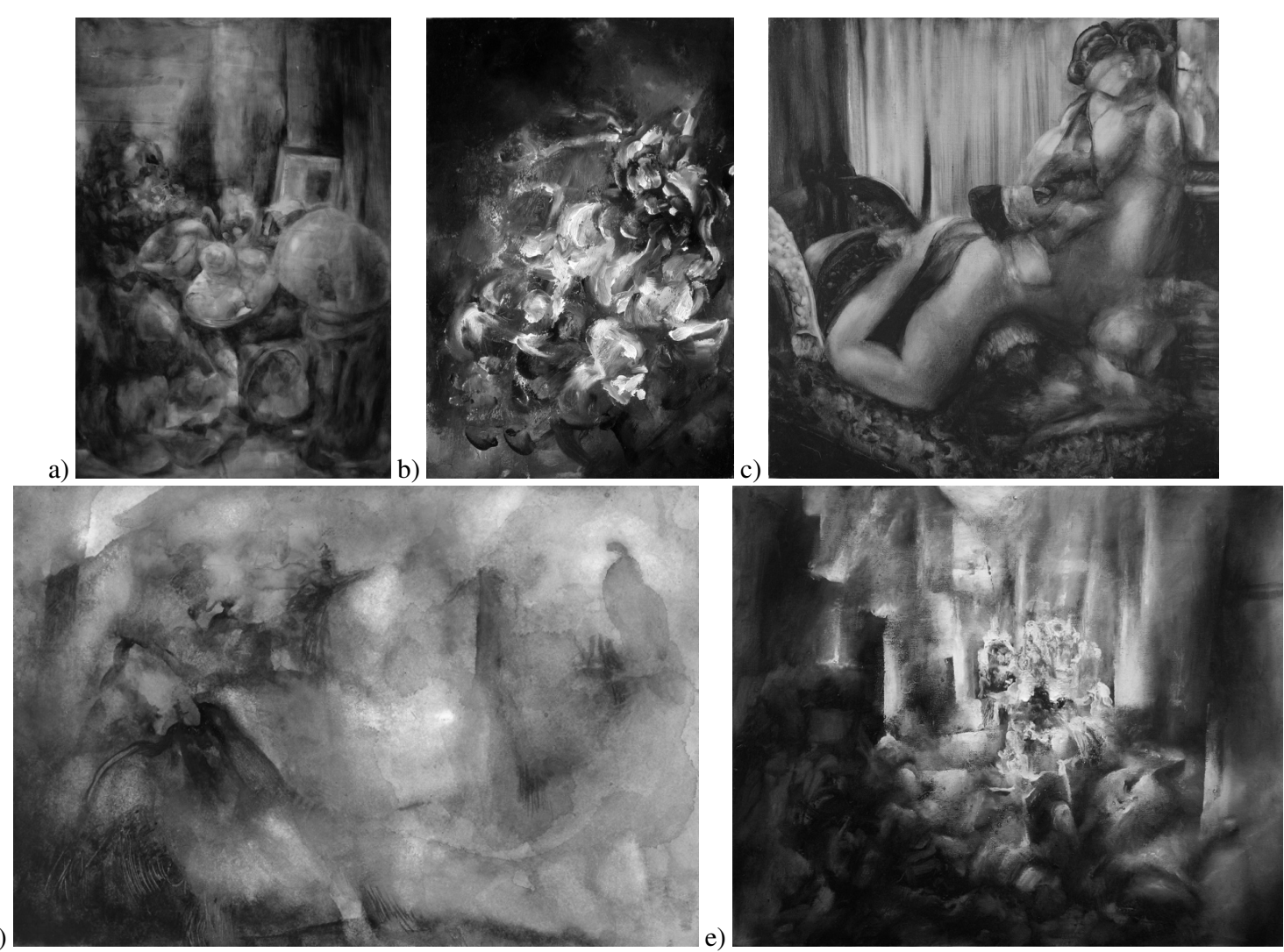

Figure 8: Genre categorization: The three most a) uniquely and the two most b) ambiguously classified images. 